\title{
Quantitative evaluation of powder spray effects on stereovision measurements
}

\author{
Zhenhua Huang, Jun Ni and Albert J Shih \\ Mechanical Engineering, University of Michigan, Ann Arbor, MI 48109, USA \\ E-mail: shiha@umich.edu \\ Received 4 June 2007, in final form 12 November 2007 \\ Published 14 January 2008 \\ Online at stacks.iop.org/MST/19/025502
}

\begin{abstract}
The effects of powder spray on surface topography and gauge repeatability and reproducibility $(\mathrm{R} \& \mathrm{R})$ of a stereovision measurement system are investigated for specular machined components. Results obtained in the micro-scale laser profilometer and macro-scale stereovision measurement of powder sprayed surfaces are correlated. Three artifacts, two step gauges and a gauge ball, all with specular surfaces, are utilized to evaluate this optical, non-contact measurement method. While the use of powder spray has demonstrated the reduction of the measurement noise of the stereovision system, as shown in this study, the surface topography of powder spray is not suitable for $\mu \mathrm{m}$-level precision measurements with the powder droplets as high as $98.4 \mu \mathrm{m}$. The accuracy and gauge $\mathrm{R} \& \mathrm{R}$ of the system for a $32 \mu \mathrm{m}$ step height are 9.4 and $4.6 \mu \mathrm{m}$, respectively. A case study of flatness measurement of an automotive engine head combustion deck surface is conducted.
\end{abstract}

Keywords: powder spray, stereovision measurement, machined components

(Some figures in this article are in colour only in the electronic version)

\section{Introduction}

Three-dimensional (3D) non-contact optical measurement technologies have progressively advanced and been widely utilized in quality inspection, reverse engineering, remote sensing and robot guidance [1-4]. Optical measurement systems have been applied in automotive quality control and process diagnosis of body manufacturing and assembly. Based on a recent study by Hammett et al [5], the 3D stereovision optical measurement system investigated herein, the CogniTens Optigo 200 with a field of view of $260 \mathrm{~mm} \times$ $190 \mathrm{~mm} \times 150 \mathrm{~mm}$, has the gauge repeatability and reproducibility ( $R \& R$ ), denoted as $\sigma_{\mathrm{R} \& \mathrm{R}}$, of $0.048,0.034$ and $0.039 \mathrm{~mm}$ for the hole position, surface point and edge point feature measurements of automotive body parts. The stereovision system has demonstrated its capability for $50 \mu \mathrm{m}$ level precision measurements of automotive body parts without powder spray.

Automotive powertrain components, such as engine heads and blocks, automatic transmission valve bodies and fuel cell bipolar plates, typically have more stringent dimensional and geometrical form tolerances and surface roughness requirements than body parts. The current practice for optical measurement of precision parts with specular machined surfaces requires the use of powder spray, which is commonly used in practical measurement applications. However, the effect of powder spray on precision optical measurement has not been quantified.

The coordinate measuring machine (CMM), first developed in the 1950s [6], is a flexible and accurate inspection tool for automotive powertrain components. CMM does not require powder spray if the contact-based probe is applied. However, due to the extended measurement cycle and limited surface coverage, CMM is not feasible for the inline inspection desired for automotive powertrain component transfer line production applications.

Several 3D optical measurement systems have been developed and are commercially available in the market. Commonly used optical measurement systems can be classified as laser triangulation [8], structured lighting $[9,10]$, stereovision [11], moiré fringe [12,13], interferometry [14], focus detection [15], time-of-flight [16], echography [17], nuclear magnetic resonance [17] and tomography [17]. There is still a lack of measurement standards to evaluate optical measurement systems. The goal of this research is to 
explore the use of various artifacts and procedures and to study the effect of powder spray on the stereovision measurements.

The stereovision measurement system is selected for three reasons. First, it is capable of non-contact full surface measurement and forming a digital representation of large objects with complicated shape $[18,19]$. Second, the system can acquire images at the microsecond level, which can reduce the measurement time for in-line applications. Third, it is a flexible measurement system and can be quickly and easily reconfigured for parts of different size, shape and family.

In this paper, the laser profilometer and stereovision measurements of powder sprayed surfaces are presented. The artifacts, measurement procedures and data analysis to evaluate the accuracy and precision of a stereovision measurement system are described. Measurement results and statistical analysis are discussed. Effects of powder spray on the stereovision measurement of specular surfaces are analyzed.

\section{Experiment setup and procedures}

The stereovision system used in this study has three CCD camera sensors. The object is placed in the center of the field of view. The view angle between the normal of the object surface planes and the line of view, as recommended by the equipment provider, is between $20^{\circ}$ and $30^{\circ}$. The stereovision system was calibrated by calculating the trilinear tensor imaging parameters from a 3D image of the specially designed retroreflective dot targets with certified dimensions. This calibration is recommended by the system supplier to assure the best accuracy. The powder spray, measurement artifact design, procedures and data processing are discussed in the following three sections.

\subsection{Powder spray}

The powder spray used in this study is Spotcheck SKD-S2, manufactured by Magnaflux. It is a white powder chemical spray commonly used in optical measurements to reduce the surface reflectivity. The chemical in liquid form is delivered from an aerosol can and atomized into small droplets, which fall and adhere to the surface and solidify into powder.

Two methods of applying powder spray, called sparse and dense spray, are experimented with. In sparse spray, a small portion of the original surface remains visible after applying the spray. In dense spray, the whole surface is covered by the powder spray and no original surface is visible. A noncontact laser profilometer is used to evaluate the topography of sparsely and densely sprayed surfaces.

\subsection{Measurement artifacts}

Two four-step gauges and a gauge ball, all made of stainless steel, are used as artifacts to evaluate the dimensional and geometrical measurement accuracy and precision of a 3D stereovision system. Step gauge \#1, as shown in figure 1(a), has three ground step heights. The ground surface is specular with about $0.2 \mu \mathrm{m} R_{a}$ roughness. Half of step

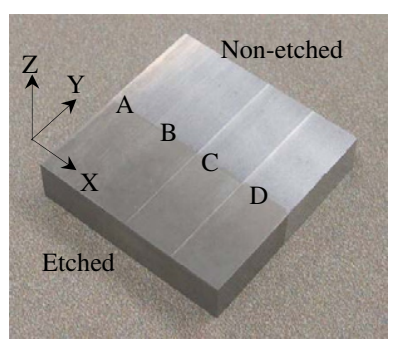

(a)

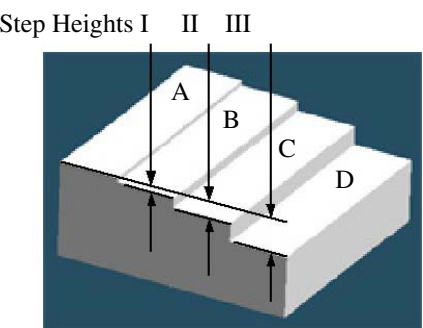

(c)

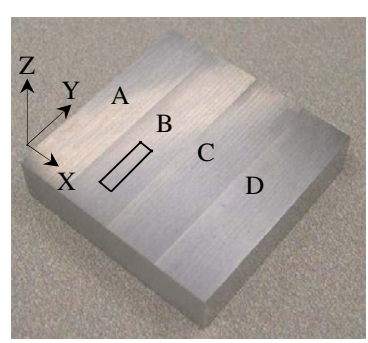

(b)

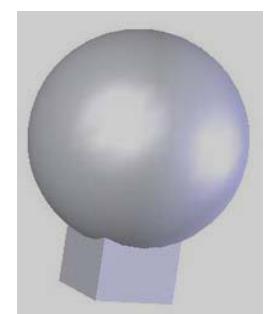

(d)
Figure 1. Three artifacts: (a) half-etched step gauge \#1, $(b)$ step gauge \#2 with no etching, $(c)$ three step heights of the step gauge and $(d)$ gauge ball.

gauge \#1 is chemically etched in an ethanol solution with $6.3 \mathrm{wt} \%$ of $\mathrm{FeCl}_{3}$ and $7.5 \mathrm{wt} \%$ of $\mathrm{HCl}$ for $30 \mathrm{~s}$ to reduce the surface reflectivity. The surface roughness remains at about $0.2 \mu \mathrm{m} R_{a}$ but the reflectivity is reduced significantly. The half-etched step gauge \#1 has eight surfaces, four of which are chemically etched surfaces and the other four are original ground surfaces, as indicated in figure 1(a). The area of each surface segment in figure $1(a)$ is $12 \mathrm{~mm} \times 24 \mathrm{~mm}$. Step gauge \#2, as shown in figure $1(b)$, has four precisely ground surfaces. As shown in figures $1(a)-(c)$, four ground surfaces are denoted as A, B, C and D. Both step gauges have about $48 \mathrm{~mm} \times 48 \mathrm{~mm}$ measurement surface area. Three step heights, designated as I, II and III in figure $1(c)$, are used to evaluate the stereovision measurement system. The nominal values of the three step heights I, II and III are 5, 15 and $35 \mu \mathrm{m}$, respectively. Such small step heights are used to test the performance limitations of the stereovision measurement system. Etching does not change the step height significantly, as will be shown later in the measurement results.

The precision stainless steel gauge ball, as shown in figure $1(d)$, is used to evaluate the precision form measurement. The size of the ball is $14.288 \pm 0.003 \mathrm{~mm}$ in diameter. The sphericity of the ball is less than $0.6 \mu \mathrm{m}$.

\subsection{Artifacts measurement procedures}

For step gauge \#1, two groups of measurements, one with and the other without the powder spray, are conducted to investigate effects of surface reflectivity and powder spray. Each group has 11 independent 3D measurements. The powder spray remains unchanged during the 11 measurements. The relative location between the sensors and the artifact also remains unchanged in each measurement group. For step gauge \#2, three groups, each with 11 measurements, are 
conducted to analyze the accuracy and $\sigma_{\mathrm{R} \& \mathrm{R}}$ of the stereovision measurement system.

For the precision ball gauge, three groups of $113 \mathrm{D}$ measurements are conducted with the powder spray. The sparse powder spray is applied at the start of each group of measurements. The ball is not moved and the spray is not changed through the 11 measurements.

\subsection{Data analysis}

Each 3D image capturing generates a point cloud. Each point has $X, Y$ and $Z$ coordinate data. For step gauge \#1, eight rectangular segments are selected to represent the etched and non-etched surface areas. Each of the $12 \mathrm{~mm} \times 24 \mathrm{~mm}$ surface segments has about 1200 data points. For step gauge \#2, four rectangular segments are identified to represent surfaces A, B, $\mathrm{C}$ and $\mathrm{D}$.

2.4.1. Step height. The least-squares method is used to calculate the step height between two segments of data points. Three step heights I, II and III, as shown in figure 1(b), are referenced from the datum surface $\mathrm{A}$. The points representing surface $J$ are denoted as the vector $\boldsymbol{P}_{J i}$, where $J=\mathrm{A}, \mathrm{B}, \mathrm{C}$ and $\mathrm{D}, i=1,2, \ldots, n_{J}$, and $n_{J}$ is the number of points on surface $J$. The middle point of surface $J$, denoted as $\boldsymbol{P}_{J M}$, is calculated by

$$
\boldsymbol{P}_{J M}=\frac{\sum_{i=1}^{n_{J}} \boldsymbol{P}_{J i}}{n_{J}}
$$

The vector from a point in the segment to the middle point of plane $J$ is $\boldsymbol{P}_{S i}-\boldsymbol{P}_{S M}, S=\mathrm{B}, \mathrm{C}$ and $\mathrm{D}$ and $i=1,2, \ldots, n_{S}$. Two groups of vectors $\boldsymbol{P}_{A i}-\boldsymbol{P}_{A M}$ and $\boldsymbol{P}_{S i}-\boldsymbol{P}_{S M}(S=\mathrm{B}, \mathrm{C}$ and D) are combined together and a least-square plane is calculated to fit these two combined segments. The normal vector of the least-square plane is $N$. The step height between two segments is $d=\left|\left(\boldsymbol{P}_{A M}-\boldsymbol{P}_{S M}\right) \cdot \boldsymbol{N}\right|$.

2.4.2. Linear transformation of measured points. The original point cloud generated by the stereovision measurement system is referenced to the machine coordinate system $X^{\prime} Y^{\prime} Z^{\prime}$. The point cloud data need to be transformed to a new $X Y Z$ coordinate system, as shown in figures 1( $a$ ) and (b), to view and calculate the variation of measurements. For step gauge \#1, eight sets of point cloud data are obtained on the eight etched and non-etched surfaces, as shown in figure $1(a)$. The $Z$-axis is perpendicular to the measured surface. The $Y$-axis is parallel to the line between two adjacent step surfaces. The $X$-axis is parallel to the measured surfaces and forms a right-handed coordinate system with the $Y$ - and $Z$-axes. From the measured data points, the average of four normal vectors of the least-square planes of the four measured surfaces is used to represent the $Z$-axis. These four normal vectors are very close to each other in actual measurements. To calculate the $X$-axis, the average of data points in each of the four etched surfaces (A, B, C and D) is used to calculate the representative middle points $\boldsymbol{P}_{A M}, \boldsymbol{P}_{B M}, \boldsymbol{P}_{C M}$ and $\boldsymbol{P}_{D M}$, as shown in figure 2. From these four middle points, six unit vectors between any two points can be obtained. The $Z$-axis and the average of these six vectors form a plane. The $X$-axis is on this plane and perpendicular to the $Z$-axis. Once the $X Y Z$ coordinate system

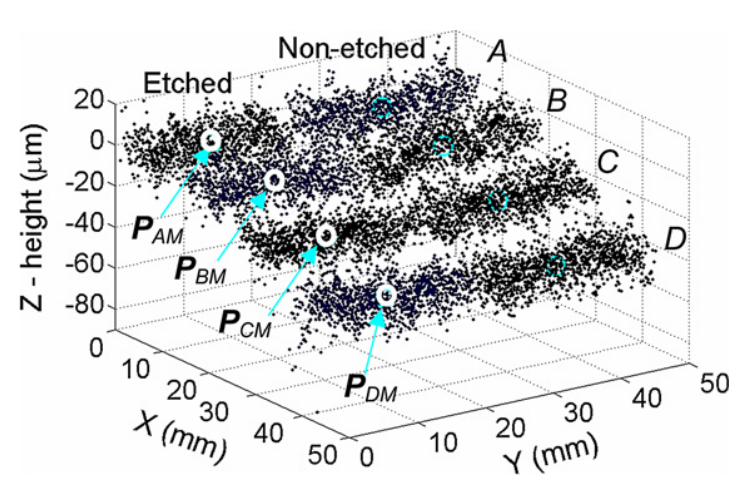

Figure 2. An example of the stereovision measured point cloud after coordinate transformation.

is defined, all the measured data points are transformed to this new coordinate system.

2.4.3. Ball diameter. For the precision ball gauge, a leastsquare sphere [20] is fitted to the point cloud to find the diameter of the ball. This diameter is compared to the measured size of the ball for accuracy evaluation.

\section{Powder spray surface topography}

Manually applied powder spraying is a random process. The variation of aerosol fluid atomization, quantity, delivery, drying and other factors can all change the topography of sprayed surfaces [21]. A laser-based profilometer, manufactured by Rodenstock, with the $10 \mathrm{~nm}$ depth and $10 \mu \mathrm{m}$ lateral resolutions is used to measure the topography of sparsely and densely sprayed surfaces.

\subsection{Sparsely sprayed surface topography}

On sparsely sprayed surfaces, a portion of the original surface remains uncovered after the powder spray is applied, i.e., the powder spray does not cover the whole surface area. Figure 3(a) shows the topography of a $1 \mathrm{~mm} \times 10 \mathrm{~mm}$ sparsely sprayed rectangular area on the flat surface of step gauge \#2. This rectangular area, also outlined in figure $1(b)$, consists of two segments: $E$ and F. Segment $E$ is the $1 \mathrm{~mm} \times 2.5 \mathrm{~mm}$ originally ground surface, which was masked during spraying. The ground surface is used as datum for measuring the relative height of droplet on the sparsely sprayed surface. Segment F is the $1 \mathrm{~mm} \times 7.5 \mathrm{~mm}$ sparsely sprayed area. Several high peaks in the range of $80-100 \mu \mathrm{m}$ are observed. To better understand the surface topography, a $1 \mathrm{~mm} \times 1 \mathrm{~mm}$ closeup view of segment $\mathrm{G}$, which contains the highest peak of $98.4 \mu \mathrm{m}$, is illustrated in figure 3(b). Many peaks created by individual droplets can be identified in figure $3(b)$. Since the stereovision measurement system used in this study was set to $0.3 \mathrm{~mm}$ point spacing, two $0.3 \mathrm{~mm} \times 0.3 \mathrm{~mm}$ areas, marked as $\mathrm{H}$ and $\mathrm{I}$ in figure $3(b)$ ), are exposed for further investigation. Figure 3(c) shows the isometric view of area $\mathrm{H}$ with the highest peak. This high peak is likely due to a large droplet or several small droplets falling close to each other. Several smaller droplet peaks and the ground surface area without spray can 


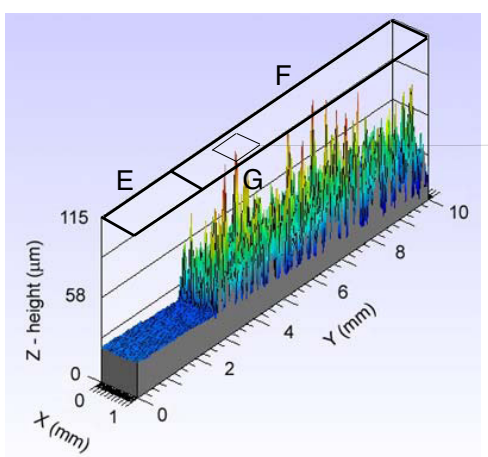

(a)

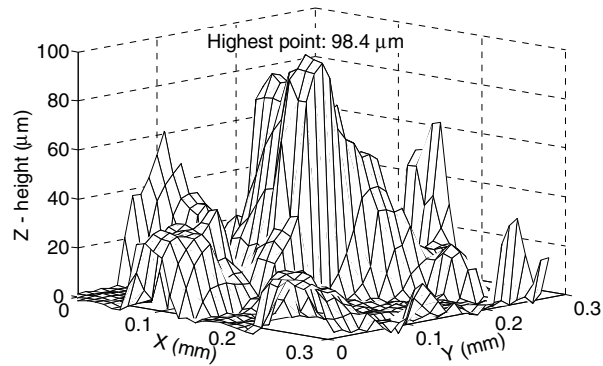

(c)

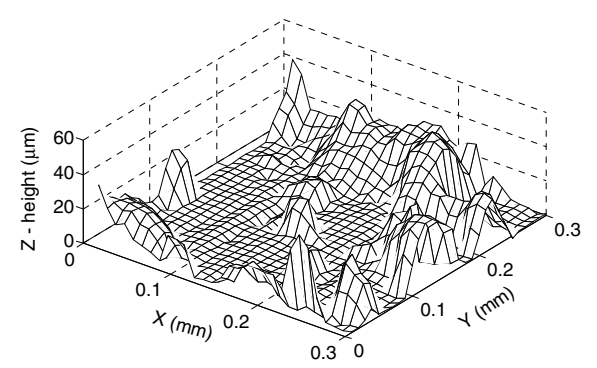

(e)

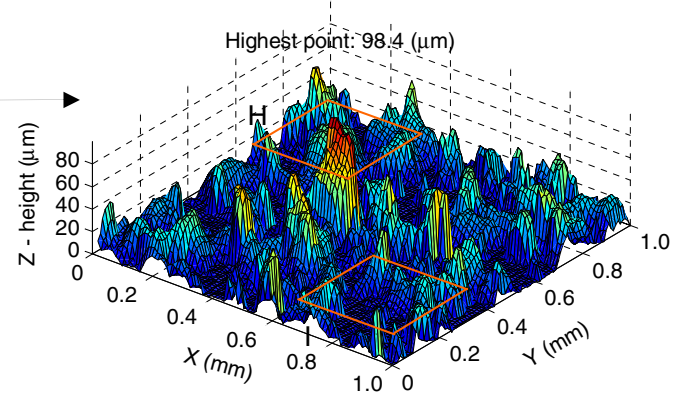

(b)

Unit: $\mu \mathrm{m}$

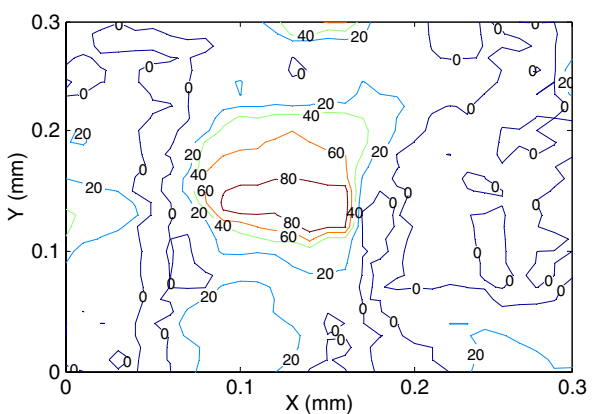

(d)

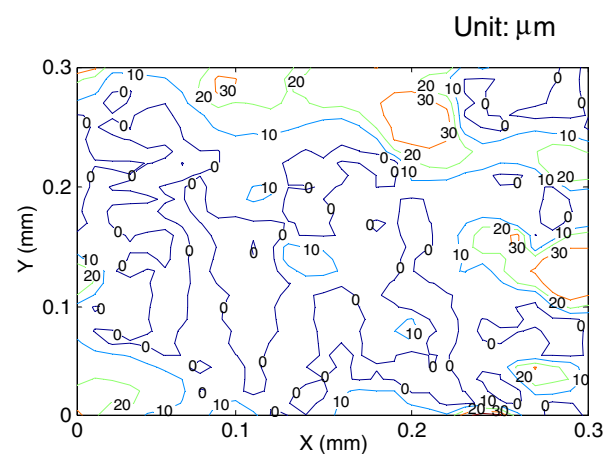

$(f)$

Figure 3. Sparsely sprayed surface topography: $(a)$ overview of the segments with and without powder spray, $(b)$ close-up view of the area $\mathrm{G}$ in $(a)$ and the highest peak, $(c)$ close-up view of area $\mathrm{H}$ in $(b)$ and the highest peak, $(d)$ contour plot of the area $\mathrm{H},(e)$ close-up view of the area $\mathrm{I}$ in $(b)$ and $(f)$ contour plot of the area I.

still be seen in this small $0.3 \mathrm{~mm} \times 0.3 \mathrm{~mm}$ area. The contour plot of this area, as shown in figure $3(d)$, validates such observation. Area I, as shown in figure 3(e), has relatively low peak height of $46.0 \mu \mathrm{m}$, produced by some small droplets. A larger portion of area I is not covered by the powder spray.

\subsection{Densely sprayed surface topography}

Under dense spray, the whole measurement surface area is covered by the white powder. No original ground surface is visible. Figure $4(a)$ shows a $1 \mathrm{~mm} \times 10 \mathrm{~mm}$ segment of densely sprayed surface. Compared to figure 3(a), a different surface topography without spike-shaped peaks is observed. A low spatial frequency waviness can also be observed in figure $4(a)$. There is no datum surface in the densely sprayed area. By fitting a least-square plane of the densely sprayed surface and setting the lowest point as zero height, the highest point on densely sprayed surface is $62.5 \mu \mathrm{m}$, which is lower than the highest peak height on sparsely sprayed surfaces. Close-up view of the $0.3 \mathrm{~mm} \times 0.3 \mathrm{~mm}$ area $\mathrm{J}$ surrounding the highest peak is shown in figure $4(b)$. The peak is relatively flat, compared to steep peaks in figures $3(a)$ and $(c)$.

\section{Artifact measurement results and statistical analysis}

Results of the stereovision measurement of step gauge \#1, step gauge \#2 and gauge ball are presented in the following three sections. 


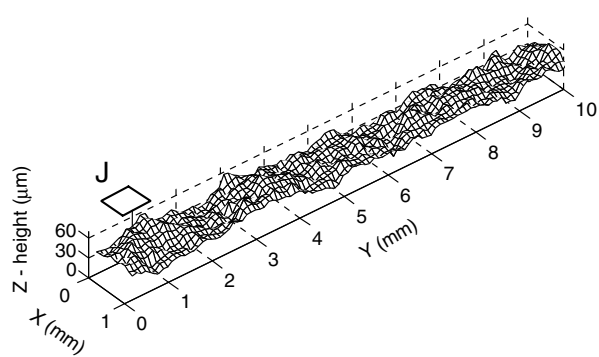

(a)

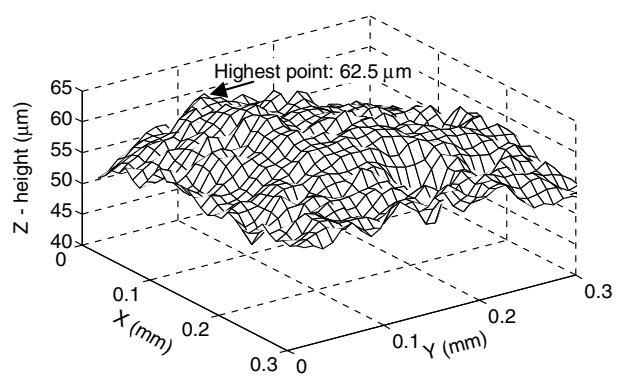

(b)

Figure 4. Densely sprayed surface topography: (a) overview and (b) close-up view of the area $\mathrm{J}$ in $(a)$.

Table 1. Plane distance measurement results for step gauge \#1.

\begin{tabular}{|c|c|c|c|c|c|c|c|c|}
\hline \multirow{2}{*}{$\begin{array}{l}\text { Measurement } \\
\text { method }\end{array}$} & \multirow{2}{*}{\multicolumn{2}{|c|}{ Step height }} & \multicolumn{3}{|c|}{ Etched } & \multicolumn{3}{|c|}{ Non-etched } \\
\hline & & & I & II & III & I & II & III \\
\hline Profilometer & & Target value & 3.9 & 17.2 & 36.8 & 3.5 & 15.4 & 35.1 \\
\hline \multirow[t]{6}{*}{ Stereovision } & Non-sprayed & $\mu_{d n}(\mu \mathrm{m})$ & 0.8 & 13.4 & 28.9 & 1.8 & 15.1 & 36.9 \\
\hline & & Discrepancy (\%) & 79 & 22 & 21 & 49 & 2 & 5 \\
\hline & & $\sigma_{d n}(\mu \mathrm{m})$ & 0.6 & 2.7 & 4.0 & 1.0 & 1.8 & 3.9 \\
\hline & Sprayed & $\mu_{d s}(\mu \mathrm{m})$ & 2.8 & 15.7 & 38.8 & 0.6 & 15.3 & 39.2 \\
\hline & & Discrepancy (\%) & 28 & 9 & 5 & 83 & 0.6 & 12 \\
\hline & & $\sigma_{d s}(\mu \mathrm{m})$ & 0.5 & 1.2 & 2.4 & 0.4 & 1.2 & 1.3 \\
\hline
\end{tabular}

$\mu_{d n}, \mu_{d s}:$ mean of step height measurement.

$\sigma_{d n}, \sigma_{d s}:$ standard deviation of step height measurement.

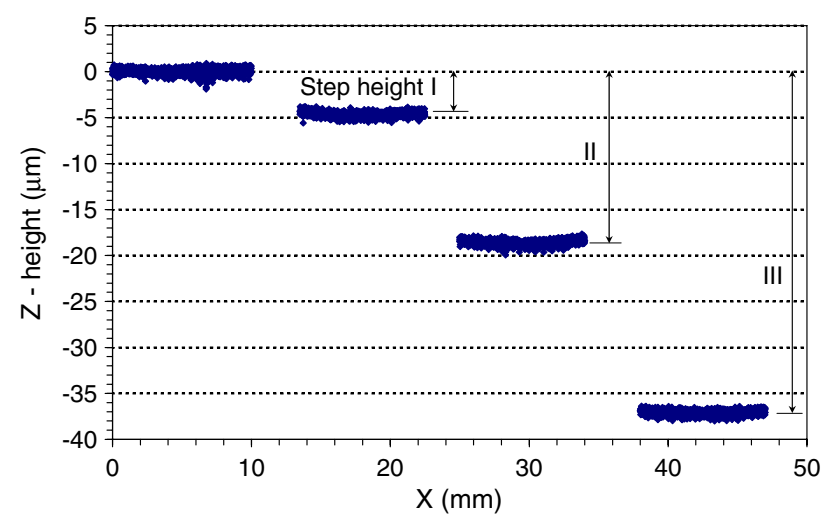

Figure 5. Target value measurement of step heights by Taylor-Hobson contact profilometer.

\subsection{Step gauge \#1}

4.1.1. Target value of step heights. The target value of step heights is measured using the Taylor Hobson Form Talysurf profilometer. Ten measurement traces, all parallel to each other and perpendicular to the line between two adjacent step surfaces, are used to measure step heights I, II and III. The etched and non-etched sides of step gauge \#1, as shown in figure 1(a), each have five measurement traces. An example of the profilometer measurement trace on the non-etched surface is shown in figure 5. The algorithm presented in section 2.3 is used to calculate the step height. The five measured step heights I range from 3.4 to $4.6 \mu \mathrm{m}$ with $3.9 \mu \mathrm{m}$ average on the etched side and from 2.7 to $4.2 \mu \mathrm{m}$ with $3.5 \mu \mathrm{m}$ average on the non-etched surfaces. The average of the five measurements is used as the target value listed in table 1 . The range of the five measurements, $1.2 \mu \mathrm{m}$ on the etched and $1.5 \mu \mathrm{m}$ on the nonetched side, is likely due to the inconsistency in grinding and etching processes. The value may be high for the shallow step height I. For step height III, the range of five measurements is 3.2 and $2.8 \mu \mathrm{m}$ for the average height (target value) of 36.8 and $35.1 \mu \mathrm{m}$ on the etched and non-etched sides, respectively. The range of measurement variation relative to the target value is much smaller for Step Height III, which is used for more reliable evaluation of $\sigma_{\mathrm{R} \& \mathrm{R}}$.

4.1.2. Step height measurement. Two groups of 11 measurements, one group with and the other without powder spray, are conducted. From the 11 measured step heights without powder spray, the mean value, $\mu_{d n}$, its discrepancy with the target value, and the standard deviation, $\sigma_{d n}$, for the etched and non-etched surfaces are calculated and summarized in table 1.

Comparing $\mu_{d n}$ with the target value, it has a discrepancy in step height I, $79 \%$ for the etched and $49 \%$ for the nonetched surfaces. For step heights II and III, the discrepancies are much smaller. More in-depth analysis of the accuracy in step height measurement will be discussed in section 4.2, step gauge \#2.

The effect of powder spray on the discrepancy of height measurement for step heights II and III is mixed. For the step height II, the application of powder spray reduces the step height measurement discrepancy. However, for the step height III of non-etched surfaces, the discrepancy of height measurement increases from $5 \%$ to $12 \%$ with powder spray. 


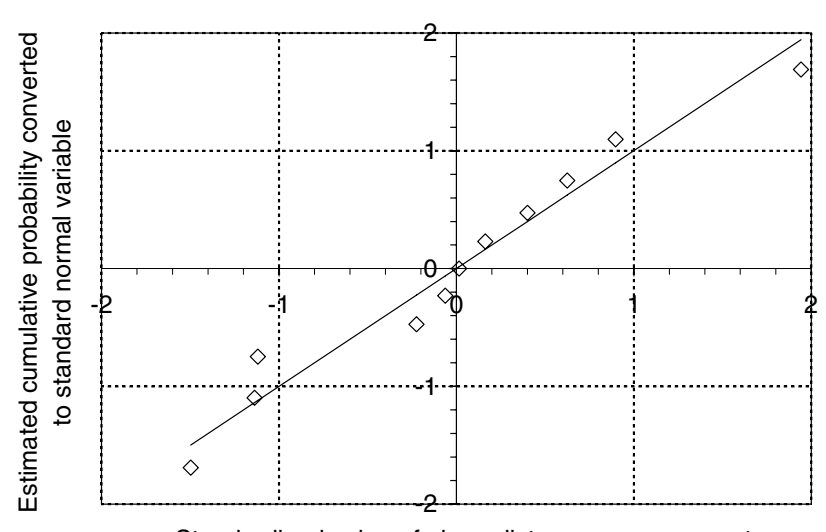

Standardized value of plane distance measurement

Figure 6. An example of the $Q-Q$ plot for 11 measurements of etched step height III of the step gauge \#1 with powder spray.

The statistical analysis is applied to identify effects of surface reflectance (etched versus non-etched) and powder spray (sprayed versus non-sprayed) on step height measurements.

The $F$-test [22] is applied to compare the step height measurement variance between the non-etched and etched surfaces. Results for step heights II and III are 1.07 and 0.27 for non-sprayed and 0.44 and 0.91 under the sprayed condition. All results are within the $95 \%$ critical region $[0.27,3.72]$. This means that, with $95 \%$ confidence, the etched step height measurement is not expected to be more repeatable than non-etched surfaces under both sprayed and non-sprayed conditions. The $Q-Q$ plot was applied to verify if the 11 measurements in each test follow the normal distribution [23]. Figure 6 shows an example of the $Q-Q$ plot for the 11 step height I measurements of step gauge \#1. All 11 discrete points are close to the $45^{\circ}$ line, which demonstrates that it can be justified to follow the normal distribution. The other measurement groups of step heights II and III have similar trend in $Q-Q$ plots.

The $F$-test is also applied to compare the variance in step height measurement between non-sprayed and sprayed surfaces. The $F$-test results are 2.11 and 9.23 for nonetched and 5.17 and 2.77 for etched step heights II and III, respectively. One of the two non-etched step heights and one of the two etched step heights have the $F$-test beyond the $95 \%$ critical region $[0.27,3.72]$. This mixed result indicates that the powder spray does not necessarily make height measurement more repeatable.

The $t$-test [22] is applied to check if powder spray can improve the accuracy in step height measurement. The $t$ test results are 0.26 and 1.83 for non-etched and 2.60 and 6.94 for etched step heights II and III, respectively. Two etched surfaces have $t$-test values beyond the corresponding $95 \%$ critical region and two non-etched surfaces have $t$-test values in the corresponding $95 \%$ critical region. This mixed result indicates that the powder spray does not necessarily make height measurement more accurate.

From these observations, it can be concluded that the powder spray does not necessarily improve the performance of step height measurement by the stereovision system. It should also be pointed out that, as the surface area increases, the uniformity of the powder spray thickness may be limited and the relative plane distance could vary.

4.1.3. Variation of measurements. For each measured surface, the standard deviation of the distances from measured data points to the least-square plane, denoted as $\sigma_{v}$, is calculated. This $\sigma_{v}$ represents the quantitative evaluation of the system measurement noise because the surface after grinding is flat less than $1 \mu \mathrm{m}$ from the measurement of segment $\mathrm{E}$ in figure $3(a)$. The non-etched surface, measured using the laser-based Rodenstock Model RM600 profilometer, has about $1 \mu \mathrm{m} \sigma_{v}$. Much higher $\sigma_{v}$ is observed in stereovision measurements. Without powder spray, an example of the side view ( $X Z$ plane) of four etched and four non-etched surfaces of step gauge \#1 is shown in figures $7(a)$ and $(b)$. The $\sigma_{v}$ is large, 10.8-14.5 $\mu \mathrm{m}$ for etched and 7.0-8.9 $\mu \mathrm{m}$ for non-etched surfaces. Figure 7 shows only one of the 11 measurements. The mean and standard deviation of $\sigma_{v}$ for 11 non-sprayed measurements, denoted as $\mu_{\sigma_{V}, n}$ and $\sigma_{\sigma_{V}, n}$, respectively, are listed in table 2 .

Figures $7(c)$ and $(d)$ show an example of one of the 11 measurements for surfaces with the powder spray. $\sigma_{v}$ is smaller under the spray condition: $5.7-6.4 \mu \mathrm{m}$ for etched and 5.8-7.2 $\mu \mathrm{m}$ for non-etched surfaces. The effect of powder spray to reduce $\sigma_{v}$ can be identified by comparing the variation of data points in figures $7(c)$ and $(d)$ with figures $7(a)$ and $(b)$. The mean and standard deviation of $\sigma_{v}$ for the 11 sprayed measurements, denoted as $\mu_{\sigma_{V}, s}$ and $\sigma_{\sigma_{V}, s}$, respectively, are also listed in table 2.

Table 2 shows that the mean of $\sigma_{v}$ with powder spray $\left(\mu_{\sigma_{V}, s}\right)$ is reduced by $4.5-7.2 \mu \mathrm{m}$ for the four etched and $0.3-1.6 \mu \mathrm{m}$ for the four non-etched surfaces when compared with the mean of $\sigma_{v}$ without powder spray $\left(\mu_{\sigma_{V}, n}\right)$. The standard deviation of $\sigma_{v}$ with powder spray $\left(\sigma_{\sigma_{V}, s}\right)$ is reduced by $0.35-1.80 \mu \mathrm{m}$ for the four etched and $0.06-0.21 \mu \mathrm{m}$ for the four non-etched surfaces. The $F$-test is applied to compare $\sigma_{\sigma_{V}, s}$ with $\sigma_{\sigma_{V}, n}$ of each surface. The $F$-test results are 72.2, $22.4,65.5$ and 863 for the etched and 11.3, 1.85, 16.0 and 7.88 for the non-etched planes A, B, C and D, respectively. All the results except one are beyond the $95 \%$ critical region $[0.27,3.72]$. This means that, with $95 \%$ confidence, the plane variation measurement using stereovision system is expected to be more repeatable if powder spray is properly applied. The $t$-test is applied to compare $\mu_{\sigma_{V}, s}$ with $\mu_{\sigma_{V}, n}$. The $t$-test results are $30.8,33.7,33.5$ and 11.2 for the etched and 17.6, 13.6, 16.5 and 4.35 for the non-etched planes A, B, C and D, respectively. All the results are beyond the corresponding $95 \%$ critical region. With $95 \%$ confidence, the $\sigma_{v}$ measurement under the properly sprayed condition is expected to be more reliable than without powder spray for both etched and nonetched planes. This concludes that the powder spray, when applied properly, can reduce $\sigma_{v}$, the measurement noise of the stereovision system on specular machined surfaces.

\subsection{Step gauge \#2}

The accuracy and $\sigma_{\mathrm{R} \& \mathrm{R}}$ of the step height measurement are evaluated using step gauge \#2. The sparse powder spray is 


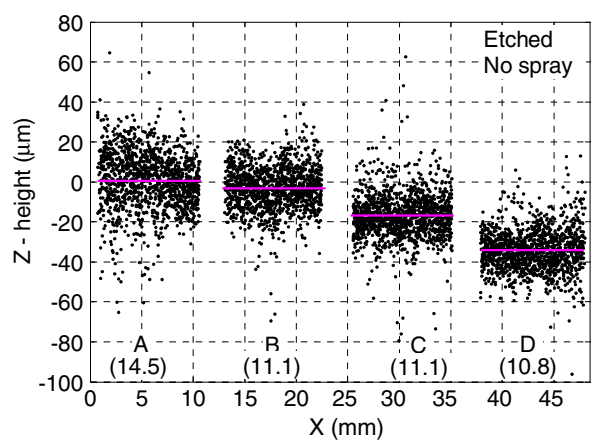

(a)

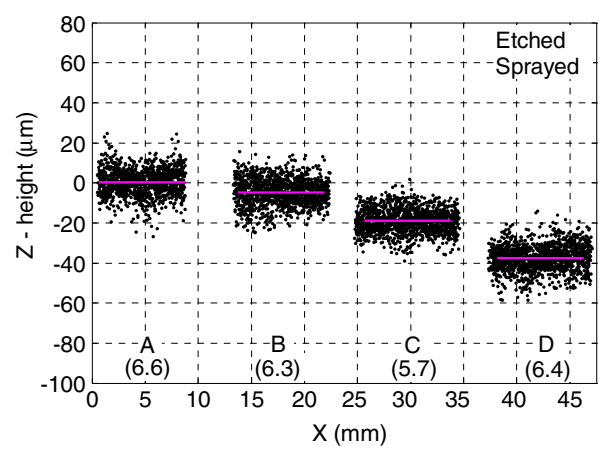

(c)

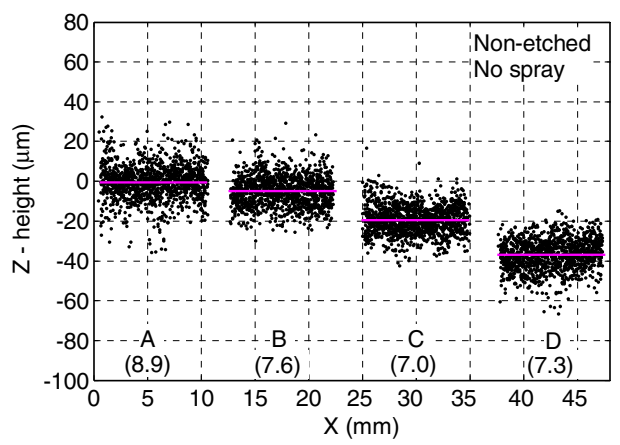

(b)

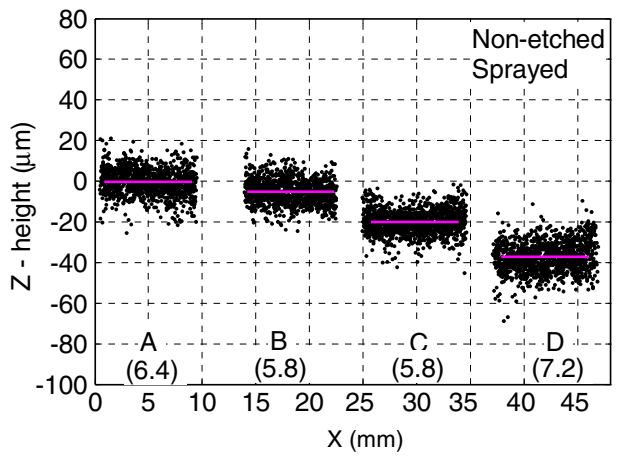

(d)

Figure 7. Side view and variation of point cloud on planes in step gauge \#1: $(a)$ etched planes without powder spray, $(b)$ etched planes with powder spray, $(c)$ non-etched planes without powder spray and $(d)$ non-etched planes with powder spray. (Standard deviations of distances from the surface data points to the least square plane, in $\mu \mathrm{m}$, are given in parentheses.)

Table 2. Plane variation $\sigma_{v}$ measurement results of step gauge \#1 (unit: $\mu \mathrm{m}$ ).

\begin{tabular}{|c|c|c|c|c|c|c|c|c|c|}
\hline & & \multicolumn{4}{|c|}{ Etched planes } & \multicolumn{4}{|c|}{ Non-etched planes } \\
\hline & & $\mathrm{A}$ & B & $\mathrm{C}$ & $\mathrm{D}$ & $\mathrm{A}$ & $\mathrm{B}$ & $\mathrm{C}$ & $\mathrm{D}$ \\
\hline \multirow[t]{2}{*}{ Non-sprayed } & $\mu_{\sigma_{V}, n}$ & 13.6 & 10.8 & 10.7 & 12.7 & 8.1 & 7.3 & 6.9 & 7.5 \\
\hline & $\sigma_{\sigma_{V}, n}$ & 0.77 & 0.44 & 0.49 & 1.86 & 0.3 & 0.25 & 0.22 & 0.24 \\
\hline \multirow[t]{2}{*}{ Sprayed } & $\mu_{\sigma_{V}, s}$ & 6.4 & 6.3 & 5.7 & 6.4 & 6.5 & 6.0 & 5.7 & 7.2 \\
\hline & $\sigma_{\sigma_{V}, s}$ & 0.09 & 0.09 & 0.06 & 0.06 & 0.09 & 0.19 & 0.05 & 0.08 \\
\hline
\end{tabular}

$\mu_{\sigma_{V}, n}, \mu_{\sigma_{V}, s}:$ mean of $\sigma_{v}$.

$\sigma_{\sigma_{V}, n}, \sigma_{\sigma_{V}, s}:$ standard deviation of $\sigma_{v}$.

applied through three measurement groups. Step gauge \#2 is not etched and the area of each step surface is $12 \mathrm{~mm} \times$ $48 \mathrm{~mm}$, twice the measurement surface of step gauge \#1. The target value of the step height is measured by the Taylor Hobson Form Talysurf profilometer. As shown in table 3, target values for step heights I, II and III are equal to 3.9, 13.6 and $32.1 \mu \mathrm{m}$, respectively.

Three groups, each with 11 measurements, are conducted. The mean values, marked as $\mu_{d}$, of step heights I, II and III for three measurement groups are listed in table 3 . The deviation of $\mu_{d}$ from the target value is defined as the accuracy of the measurement system [24]. As listed in table 3, the worst accuracies in the three measurement groups for step heights I, II and III are 1.6, 2.8 and $9.4 \mu \mathrm{m}$ or $41 \%, 21 \%$ and $29 \%$ of the target value, respectively.

The $\sigma_{R \& R}$ is another index to estimate the measurement system capability. The repeatability, denoted as $\sigma_{\text {repeatability }}$ to indicate the variation within each measurement group, is estimated by [25]

$$
\sigma_{\text {repeatability }}=\frac{\sum_{i=1}^{3} R_{i} / 3}{d_{2}}
$$

where $R_{i}$ is the range of measured step heights for each group, $d_{2}$ is a constant dependent on the number of measurements in each group. In this study, the number of measurements in each group is 11 and $d_{2}=3.173$ [22]. As shown in table 3, the estimated $\sigma_{\text {repeatability for step heights I, II and III is } 0.5,0.9}$ and 1.7 , respectively.

From three different group measurements, the reproducibility of the system, denoted as $\sigma_{\text {reproducibility }}$ to indicate the variation among different measurement group means, can be estimated by [25]

$$
\sigma_{\text {reproducibility }}=\sqrt{\left(\frac{R}{d_{2}}\right)^{2}-\frac{\sigma_{\text {repeatability }}^{2}}{n}}
$$




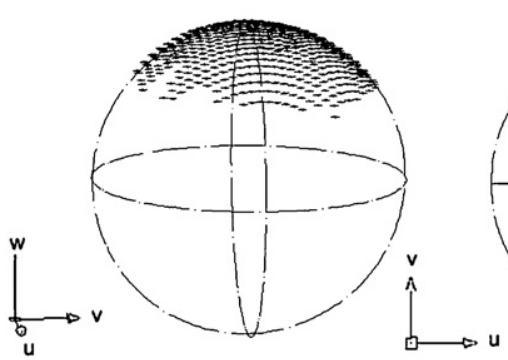

(a)

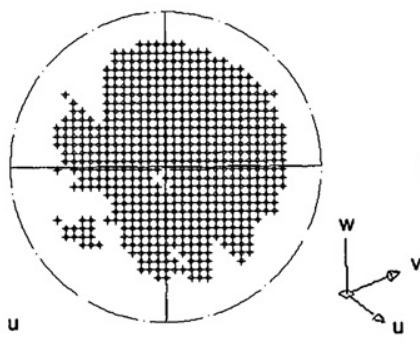

(b)

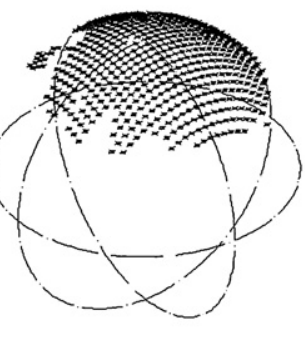

(c)

Figure 8. An example of measured point cloud on the gauge ball with powder spray: $(a)$ side view, $(b)$ top view and $(c)$ isometric view.

Table 3. Plane distance measurement results for step gauge \#2.

\begin{tabular}{|c|c|c|c|c|c|c|c|c|c|c|}
\hline \multirow{2}{*}{$\begin{array}{l}\text { Measurement } \\
\text { method }\end{array}$} & & \multicolumn{9}{|c|}{ Step height } \\
\hline & & \multicolumn{3}{|c|}{ I } & \multicolumn{3}{|c|}{ II } & \multicolumn{3}{|c|}{ III } \\
\hline Profilometer & Target value $(\mu \mathrm{m})$ & & 3.9 & & & 13.6 & & & 32.1 & \\
\hline \multirow[t]{10}{*}{ Stereovision } & Group & 1 & 2 & 3 & 1 & 2 & 3 & 1 & 2 & 3 \\
\hline & Mean, $\mu_{d}(\mu \mathrm{m})$ & 4.2 & 5.5 & 3.6 & 12.5 & 16.4 & 13.4 & 22.7 & 30.0 & 28.1 \\
\hline & Accuracy $(\mu \mathrm{m})$ & 0.3 & 1.6 & 0.3 & 1.1 & 2.8 & 0.2 & 9.4 & 2.1 & 4.0 \\
\hline & Worst accuracy among three groups $(\mu \mathrm{m})$ & & 1.6 & & & 2.8 & & & 9.4 & \\
\hline & Percentage of target value $(\%)$ & & 41 & & & 21 & & & 29 & \\
\hline & $\sigma_{\text {repeatability }}(\mu \mathrm{m})$ & & 0.5 & & & 0.9 & & & 1.7 & \\
\hline & $\sigma_{\text {reproducibility }}(\mu \mathrm{m})$ & & 1.1 & & & 2.3 & & & 4.3 & \\
\hline & $\sigma_{\mathrm{R} \& \mathrm{R}}(\mu \mathrm{m})$ & & 1.2 & & & 2.5 & & & 4.6 & \\
\hline & Smallest $\sigma_{\text {part }}$ for $\sigma_{\mathrm{R} \& \mathrm{R}} / \sigma_{\text {part }}<30 \%(\mu \mathrm{m})$ & & 4.0 & & & 8.3 & & & 15.3 & \\
\hline & $5.15 \sigma_{\text {part }}(\mu \mathrm{m})$ & & 20.6 & & & 42.7 & & & 78.9 & \\
\hline
\end{tabular}

$\mu_{d}$ : mean of step height.

$\sigma_{d}$ : standard deviation of step height.

where $R$ is the range of the three measurement group means, $d_{2}$ is a constant dependent on the number of measurement groups and $n$ is the number of tests in each group. In this study, $n=$ 11 and $d_{2}=1.693$ [22] for the group number of 3 . As shown in table 3 , the estimated $\sigma_{\text {reproducibility }}$ for step heights I, II and III are 1.1, 2.3 and 4.3, respectively.

From the estimated repeatability and reproducibility, $\sigma_{\mathrm{R} \& \mathrm{R}}$ is calculated by [25]

$$
\sigma_{\mathrm{R} \& \mathrm{R}}=\sqrt{\sigma_{\text {repeatability }}^{2}+\sigma_{\text {reproducibility }}^{2}} .
$$

For step heights I, II and III, the estimated $\sigma_{\mathrm{R} \& \mathrm{R}}$ are 1.2, 2.5 and $4.6 \mu \mathrm{m}$, respectively, as shown in table 3 .

The ratio $\sigma_{\mathrm{R} \& \mathrm{R}} / \sigma_{\text {part }}$ is used to assess the measurement system capability, where $\sigma_{\text {part }}$ is the part standard deviation. For a capable measurement system, $\sigma_{\mathrm{R} \& \mathrm{R}} / \sigma_{\text {part }}$ should be generally smaller than $30 \%$ [24]. As shown in table 3, the smallest $\sigma_{\text {part }}$ for $\sigma_{\mathrm{R} \& \mathrm{R}} / \sigma_{\text {part }}$ to be smaller than $30 \%$ are 4.0 , 8.3 and $15.3 \mu \mathrm{m}$ for step heights I, II and III, respectively. The $5.15 \sigma_{\text {part }}$, which is commonly used to cover $99 \%$ population of measurements, are 20.6, 42.7 and $78.9 \mu \mathrm{m}$, respectively. Comparing these values with the step heights, the state-ofthe-art stereovision system used in this study is not capable of separating the gauge variation from the part variation for precision step height measurement. For example, for the $32.1 \mu \mathrm{m}$ step height, if the tolerance is assumed to be $10 \mu \mathrm{m}$ $\left(=5.15 \sigma_{\text {part }}\right), \sigma_{\mathrm{R} \& \mathrm{R}}$ of the system needs to be less than $0.6 \mu \mathrm{m}$ for $\sigma_{\mathrm{R} \& \mathrm{R}} / \sigma_{\text {part }}$ to be smaller than $30 \%$. The current stereovision measurement system shows much promise in approaching the required performance, but improvements in the technology are required for industrial use on automotive powertrain applications.

$\sigma_{\text {reproducibility }}$ in table 3 is obtained by considering the range of three group means as the only effect of the system variation. However, since different powder sprays are applied for each group, the estimated $\sigma_{\text {reproducibility }}$ includes the variation of both the measurement system and the powder spray. This explains why $\sigma_{\text {reproducibility }}$ is much larger than $\sigma_{\text {repeatability }}$ in table 3 .

\subsection{Gauge ball}

The precision gauge ball is utilized to study the performance of stereovision measurement system on curved surfaces. A $14.288 \mathrm{~mm}$ precision gauge ball with powder spray is evaluated. Only a portion of the spherical surface of the gauge ball can be observed and recorded by the stereovision system in each image capturing measurement. An example of the point cloud data measured in a single image capturing of the gauge ball is shown in figure 8 . Figure $8(a)$ shows the side view of the dome-shaped area, which is used to represent the sphere. The top view of the point cloud in figure $8(b)$ shows the edges and reveals that about $25 \%$ of the total spherical surface area is covered. The isometric view in figure $8(c)$ further verifies the edge condition and the lack of data points near the apex of the ball due to light reflection. 
Table 4. Exponentially height weighted area averaging for plane variation.

\begin{tabular}{llllllllllll}
\hline$\lambda$ & 0 & 1 & 2 & 4 & 5 & 6 & 7 & 8 & 9 & 10 & 1000 \\
$\sigma_{v}(\mu \mathrm{m})$ & 3.5 & 4.0 & 4.7 & 6.8 & 8.3 & 9.8 & 11.1 & 12.3 & 13.2 & 13.8 & 15.6 \\
\hline
\end{tabular}

Three groups, each with 11 measurements, are conducted. The average diameters of the three groups are 14.294, 14.275 and $14.264 \mathrm{~mm}$, respectively. By adding the powder spray to the ball surface, the diameter is expected to be slightly larger than the $14.288 \mathrm{~mm}$ gauge diameter. The two slightly lower average ball diameters (14.275 and $14.264 \mathrm{~mm}$ ) show the limitation in using the single stereovision image for precision ball size measurement. Following the same procedures presented in section 4.2, the worst accuracy among the three groups is $23.9 \mu \mathrm{m}$. $\sigma_{\mathrm{R} \& \mathrm{R}}$ for ball diameter measurement, as determined by equations (2)-(4), is $5.2 \mu \mathrm{m}$.

The stereovision ball measurement is expected to have better results if several images captured at different angles are combined to form a more complete representation of the sphere.

\section{Correlation of spray topography and stereovision measurements}

The topography of powder sprayed surfaces has been quantified in section 3 . The correlation of the spray topography and stereovision measurement is investigated using $\sigma_{v}$ of plane surface measurements.

The $1 \mathrm{~mm} \times 7.5 \mathrm{~mm}$ sparsely sprayed segment $\mathrm{F}$ can be divided into $750.3 \mathrm{~mm} \times 0.3 \mathrm{~mm}$ regions. Each region has 900 data points measured by the Rodenstock laser profilometer with $10 \mu \mathrm{m}$ spatial spacing. Statistical analysis of the representative heights for each of the 75 regions and comparison with the plane variation results obtained in stereovision measurement can show how the stereovision measurement system performs on sprayed surfaces.

The average $\sigma_{v}$ in stereovision measurement on the sparsely sprayed, non-etched surface is about 6-7 $\mu \mathrm{m}$, as shown in table 2 . If the stereovision can only see the highest peak in the $0.3 \mathrm{~mm} \times 0.3 \mathrm{~mm}$ region, $\sigma_{v}$ of the 75 peak heights is $15.9 \mu \mathrm{m}$. This represents the upper bound of the plane variation in stereovision measurement. If only the average of 900 height data points in each of the $0.3 \mathrm{~mm} \times 0.3 \mathrm{~mm}$ regions is used, $\sigma_{v}$ is $3.5 \mu \mathrm{m}$, which is the lower bound.

In reality, the stereovision system captures neither the average height (lower bound) nor the highest peak (upper bound). The surface near the high peaks is expected to have a more significant influence on the optical sensor in stereovision measurement.

A height-dependent weighting factor, $\beta_{i}$, is defined for each profilometer-measured point, $z_{i}$, to find a representative height, denoted as $Z_{s}$, of a selected region:

$$
Z_{s}=\frac{\sum_{i=1}^{N} \beta_{i} z_{i}}{\sum_{i=1}^{N} \beta_{i}}
$$

where $N$ is the total number of points in a selected region. For this application, $N=900$ for the $0.3 \mathrm{~mm} \times 0.3 \mathrm{~mm}$ region.
The goal is to find $\beta_{i}$ that will match $\sigma_{v}$ of $Z_{s}$ for the 75 regions with $\sigma_{v}$ of stereovision measurement $(6-7 \mu \mathrm{m})$. A linearly varying weighting factor was first evaluated but failed to achieve the desired match. An exponentially decaying weighting factor is then investigated:

$$
\beta_{i}=\mathrm{e}^{-\lambda\left(T-z_{i}\right) /(T-V)}
$$

where $\lambda$ is a constant, $T$ is the height of the highest peak among all points in the region, $V$ is the height of the lowest valley among all points in the region and $z_{i}$ is the height of each point in the selected region.

At the peak point, $z_{i}=T$ and $\beta_{i}=1$, i.e., the weighting factoring is always equal to 1 at the peak. At the valley, $z_{i}=V$ and $\beta_{i}=\mathrm{e}^{-\lambda}$. For $z_{i}$ between $T$ and $V$, its corresponding $\beta_{i}$ decays exponentially with respect to the height below the peak. $\lambda$ defines the decaying rate. By selecting a higher $\lambda$, the weighting factor $\beta_{i}$ at the valley and points near the valley becomes lower, which indicates that the points near the peak are more dominant to determine the representative height $Z_{s}$.

When $\lambda=0, \beta_{i}=1$ for all $z_{i}$. According to equation (5), $Z_{s}$ is the average height of all points in the region. This corresponds to the lower bound case. When $\lambda$ approaches infinity, $\beta_{i}=1$ for the peak, $\beta_{i}=0$ for the other points and $Z_{s}=T$. This is the upper bound case.

As shown in table 4, by varying $\lambda$ from 0 to 1000 , $\sigma_{v}$ gradually increases from 3.5 (lower bound) to $15.6 \mu \mathrm{m}$ (close to upper bound). When $\lambda$ is about $4, \sigma_{v}$ matches the 6-7 $\mu \mathrm{m}$ observed in stereovision measurement system. Although the height-dependent weighting factor based on height is only a hypothesis, it does show the effect of high peaks in the micro-scale surface topography on the macroscale stereovision measurements. More in-depth research is required to investigate the correlation.

\section{Case study}

The comparison of CMM and stereovision flatness measurements of an engine head combustion deck surface, as shown in figure $9(a)$, is presented as a case study. This surface area, about $400 \mathrm{~mm} \times 250 \mathrm{~mm}$ in size, is a representative precision feature for automotive powertrain components. The flatness specifications of the surface are $150 \mu \mathrm{m}$ along the $400 \mathrm{~mm}$ length, $100 \mu \mathrm{m}$ across the $250 \mathrm{~mm}$ width and $25 \mu \mathrm{m}$ over any $25 \mathrm{~mm} \times 25 \mathrm{~mm}$ area on the surface.

Figure $9(b)$ shows the measurement results using a Zeiss UPMC CARAT 850/1200 CMM with a scanning probe. The CMM measurement is perceived to be accurate and is therefore used as the target value. However, the CMM takes long programming and measurement time and can only cover a limited percentage of the whole surface area. For example, only about $10 \%$ of the surface was measured by the scanning probe in CMM in figure $9(b)$. The stereovision measurement results are shown in figure $9(c)$. A total of 21 images were 


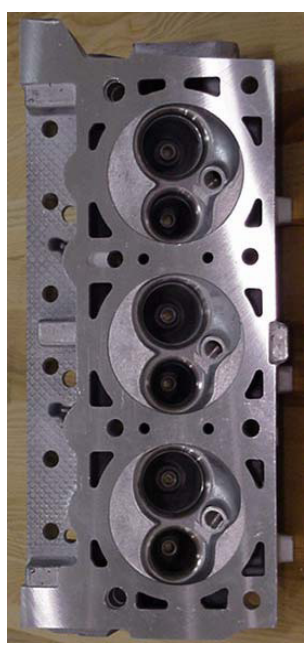

(a)

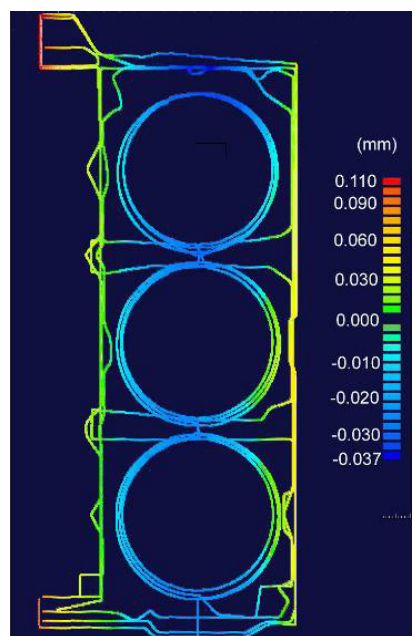

(b)

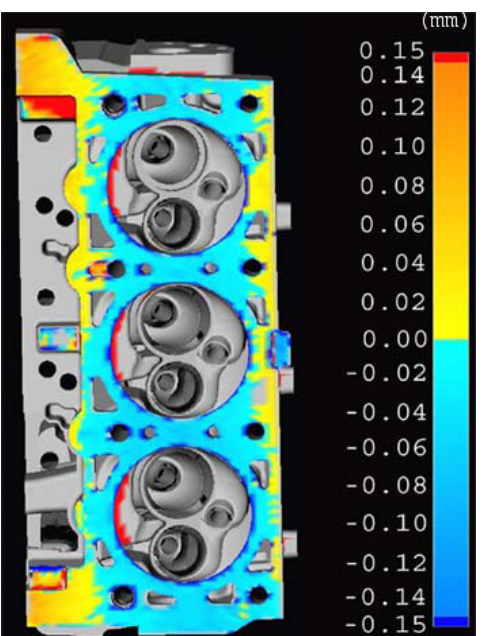

(c)

Figure 9. Comparison of the CMM and stereovision measurement of surface flatness: $(a)$ picture of the engine head combustion deck surface, $(b)$ CMM measurement results and $(c)$ stereovision measurement results.

combined to represent the whole surface. Instead of the line data in CMM, the full surface area data were obtained for flatness characterization using the stereovision system within roughly $150 \mathrm{~s}$. Compared to the CMM measured target values in figure $9(b)$, the stereovision measurement results show similar trends. The peak-to-valley flatness measured using CMM and stereovision are 150 and $190 \mu \mathrm{m}$, respectively. As observed in this study, the stereovision technology requires changes and improvement of the $\sigma_{\mathrm{R} \& \mathrm{R}}$ to meet the specifications for precision measurement in the $25 \mu \mathrm{m}$ range.

\section{Concluding remarks}

Topography of powder spray and accuracy and $\sigma_{\mathrm{R} \& \mathrm{R}}$ of a stereovision measurement system were presented. Step and ball gauges were used as the artifacts to identify the precision and accuracy of the optical measurement system. Effects of powder spray on the height measurements of specular surfaces were studied using statistical analysis methods. The correlation between the profilometer and stereovision measurements of sparsely sprayed surfaces were explored.

The measurement and statistical analysis procedures developed in this study can be applied to evaluate other optical-based measurement systems. The study shows that $\sigma_{\mathrm{R} \& \mathrm{R}}$ for the $32 \mu \mathrm{m}$ step height plane distance measurement under the sparse powder spray is $4.6 \mu \mathrm{m}$. The minimal $5.15 \sigma_{\text {part }}$ equals $78.9 \mu \mathrm{m}$, i.e., the tolerance of the $32 \mu \mathrm{m}$ step height has to be equal or larger than $78.9 \mu \mathrm{m}$ to qualify the measurement system under this measurement condition. These results demonstrate the viability of the technology, while identifying that further technical developments in both optics and algorithms are necessary to industrialize the solution for automotive powertrain applications.

The commonly used powder spray is not recommended for precision optical measurement. Though powder spray does reduce the measurement noise of a stereovision system on specular surfaces, it does not necessarily improve the height measurement accuracy or repeatability of the stereovision system. Under the sparsely sprayed condition, the height of the highest peak of the powder spray is nearly $100 \mu \mathrm{m}$, much larger than the specifications for precision automotive powertrain components. New approaches in illumination and optics are required for optical measurement of specular surfaces without the powder spray [26, 27]. It should be noted that the technology is advancing fast in optical measurement and this research is a step to understand the current level of performance achievable.

\section{Acknowledgments}

This work is sponsored by the National Science Foundation Engineering Research Center of Reconfigurable Manufacturing Systems, University of Michigan. The technical guidance from Robert Waite and Bill Haukkala of DaimlerChrysler, Marion B Grant of Caterpillar, Jun Qu of Oak Ridge National Laboratory and Guy Blumberg and Eyal Mizrahi of CogniTens is highly appreciated.

\section{References}

[1] Bradley C 1998 Computer vision techniques in reverse engineering Eng. Des. Autom. 4 101-12

[2] Maas H G 2002 Methods for measuring height and planimetry discrepancies in airborne laserscanner data Photogramm. Eng. Remote Sens. 68 933-40

[3] Rooks B W 1997 Vision helping the automotive industry to better customer choice Indust. Robot 24 48-51

[4] Shih A J and Huang Z 2006 Three dimensional optical measurements of porous foams J. Manuf. Sci. Eng. 128 951-9

[5] Hammett P C, Frescoln K D and Garcia-Guzman L 2003 Changing automotive body measurement system paradigms with $3 \mathrm{~d}$ non-contact measurement systems University of Michigan Transportation Research Institute Technical Report UMTRI-2003-43 
[6] Kierkegaard P 1996 Reflection properties of machined metal surfaces Opt. Eng. 35 845-57

[7] Bosch J A 1995 Coordinate Measurement Machines and Systems (New York: Dekker)

[8] Tai W C and Chang M 1996 Noncontact profilometric measurement of large-form parts Opt. Eng. 35 2730-5

[9] Kowarschik R, Kühmstedt P, Gerber J, Schreiber W and Notni G 2000 Adaptive optical three-dimensional measurement with structured light Opt. Eng. 39 150-8

[10] Quan C, He X Y, Wang C F, Tay C J and Shang H M 2001 Shape measurement of small objects using LCD fringe projection with phase shifting Opt. Commun. 189 21-9

[11] Zollne F, Matusevich V and Kowarschik R 2003 3D measurement by stereophotogrammetry Proc. SPIE 5144 $311-4$

[12] Wykes C and Morshedizadeh R 1995 Surface topography measurement using digital moiré contouring-errors and limitations Proc. Inst. Mech. Eng. B 209 317-25

[13] Xie X, Atkinson J T, Lalor M J and Burton D 1997 Effects on absolute moiré contouring by using gratings with different period and profile and rotating angles Opt. Lasers Eng. 27 $247-57$

[14] Wang L S, Jambunathan K, Dobbins B N and He S P 1996 Measurement of three-dimensional surface shape and deformations using phase stepping speckle interferometry Opt. Eng. $352333-40$

[15] Akuta T and Negishi Y 1991 Development of an automatic 3-D shape measuring system using a new auto-focusing method Measurement 9 (3) 98-103

[16] Kilpelä A, Pennala R and Kostamovaara J 2001 Precise pulsed time-of-flight laser range finder for industrial distance measurements Rev. Sci. Instrum. 72 2197-202

[17] Bernard A 1999 Reverse engineering for rapid product development: a state of the art Proc. SPIE 3835 50-63
[18] Shalom T 2003 System and method for aligning a locally-reconstructed three-dimensional object to a global coordinate system using partially-detected control points United States Patent \# 6556705

[19] Shalom T, Zelnik I and Goldberger J 2001 System and method for 'stitching' a plurality of reconstructions of three-dimensional surface features of object(s) in a scene defined relative to respective coordinate systems to relate them to a common coordinate system United States Patent \# 6201541

[20] Kåsa I 1976 A circle fitting procedure and its error analysis IEEE Trans. Instrum. Meas. 25 8-14

[21] Liu H 1999 Science and Engineering of Droplets, Fundamentals and Applications (New York: William Andrew Press)

[22] Montgomery D C 2001 Introduction to Statistical Quality Control (New York: Wiley)

[23] DeVor R E, Chang T H and Sutherland J W 1992 Statistical Quality Design and Control-Contemporary Concepts and Methods (Englewoods Cliffs, NJ: Prentice-Hall)

[24] Automotive Industry Action Group 2002 Measurement Systems Analysis Reference Manual Chrysler, Ford, General Motors Supplier Quality Requirements Task Force

[25] Liggett J V 1993 Dimensional Variation Management Handbook: A Guide for Quality, Design, and Manufacturing Engineers (Englewoods Cliffs, NJ: Prentice-Hall)

[26] Baba M, Ohtani K, Imai M and Konishi T 2001 New laser rangefinder for three-dimensional shape measurement of specular objects Opt. Eng. $4053-60$

[27] Pérard D and Beyerer J 1997 Three-dimensional measurement of specular free-form surfaces with a structured-lighting reflection technique Proc. SPIE 3204 74-80 